\title{
What are the clinicopathological features and outcomes of sporadic colorectal cancer (CRC) in an ethiopian cohort with focus on young-onset CRC?
}

\section{Introduction}

Sporadic Colorectal cancer (CRC) is the 3rd and 4th most common cancer worldwide in women and men respectively and is responsible for over 550,000 deaths per year. ${ }^{1,2}$ While the incidence of this disease is rising, mortality for this condition has improved greatly from previous decades through the use of improved diagnostic criteria, awareness of risk factors, treatment modalities and screening led reductions in advanced/emergency presentations. ${ }^{3,4}$

Of this Global rise in CRC developing nations are playing an increasingly larger role. This is thought to reflect their westernization of their diet and lifestyle in addition to baseline genetic risk. ${ }^{5}$. Such changes and the metabolic syndrome they predispose to are key risk factors in CRC pathogenesis and the adenoma-carcinoma sequence taking on average 10-15years. ${ }^{6,7}$ In western societies, $90 \%$ of CRC cases affect patients $>55$ years-old, however, it is becoming increasingly apparent that 'young-onset CRC' which is generally defined as $\leq 40$ years (although alternative definitions exist), is becoming more prevalent in developing and developed nations. ${ }^{1,8}$ Traditionally, young-onset CRC make up 7\% of western cohorts, however, this figure is complicated by the inclusion of patients with predisposing genetic syndromes (e.g. Lynch Syndrome) and comorbid illness (e.g. Inflammatory Bowel Disease). ${ }^{9}$ In addition most observations regarding young-onset $\mathrm{CRC}$ have been made using Surveillance Epidemiology and End Result Registries (SEERs) based on American and World Health Organisation (WHO) databases. Given these factors and the rarity of such cases relevance of young onset CRC and interpretation of epidemiology in third world cohorts remains debatable.

Early onset sporadic CRC differs from traditional CRC (associated precursor adenomatous polyps or a family history) and it remains to be seen whether there are significant clinical, biological and outcome related differences in the former. ${ }^{10}$ A recent systematic review has reported profound differences between the presentations of early versus traditional-onset CRC. These include a greater incidence of adverse prognostic factors such as mucinous/signet ring histology, venous and perineural invasion and higher tumour stage and grade resulting in worse outcomes. ${ }^{11}$ Furthermore, young-onset patients may have a predominance for left-sided/rectal malignancy and not the rightward shift in CRC distribution observed in traditional-onset populations as a result of screening protocols. ${ }^{12,4}$ Nevertheless findings between studies regarding young-onset disease are inconsistent partly due to the different definitions regarding what constitutes 'young' as well as infrequent reporting of such cases. As such there may be no real difference in the histopathological features or the survivability of this tumour type, but this must be explored given its increasing incidence. Currently treatment regimens for CRC do not discriminate by age of disease onset but are instead informed by patients' functional status, age, tumour characteristics, comorbid disease and choice. ${ }^{13}$

The aim of this study was therefore, to evaluate the epidemiology of $\mathrm{CRC}$ in Addis Ababa, Ethiopia to confirm if young-onset
Volume 8 Issue 3 - 2017

\author{
Dominic Worku,' Rajeev P Raghavan,' \\ Wondemagegnhu Tigeneh ${ }^{3}$ \\ 'University of Birmingham, UK \\ ${ }^{2}$ Consultant Physician, New Cross Hospital, Royal \\ Wolverhampton Trust, England \\ ${ }^{3}$ Medical Faculty, Black Lion Hospital,Addis Ababa University, \\ Ethiopia
}

Correspondence: Dominic Worku, University of Birmingham, I4 Cwrt-Ucha Terrace, Taibach Port Talbot Sa 3 Ild, United Kingdom, Tel 7780663345, Email DAWI64@student.bham.ac.uk

Received: June 12, 2017 | Published: August 04, 2017

sporadic disease is indeed prevalent, and furthermore relate its clinicopathological characteristics and outcomes with traditional traditional-onset disease.

\section{Methods}

The Tinkur Anbessa Hospital in Addis Ababa is a state run hospital and the sole cancer referral centre in Ethiopia and at present maintains the only known cancer registry. Patients who had presented within the last 9 months were identified using the oncology admission and follow-up registry. Data from identified sporadic CRC cases was collected via patient notes and discussions with the multidisciplinary team (MDT). Due to multiple coding formats only cases specifying the exact diagnosis or tumour location were accepted. All patients with suspected or confirmed hereditary CRC syndrome were excluded from the study.

All patients had been seen independently by the hospital's MDT, and in all cases a diagnosis of CRC was confirmed through colonoscopy and biopsy by a pathologist. As no onsite pathology/ laboratory department exists, the diagnosis, biochemistry and Carcinoembryonic Antigen (CEA) measurements were conducted by several outside private sources. Young-onset CRC was defined as $\leq 40$ years old with those $>40$ years old considered traditional-onset, these cut-offs were used in all analyses. In all cases details regarding duration of symptoms, mode and nature of presentation, co-morbid illness, baseline Eastern Cooperative Oncology Group (ECOG) status, family history, tumour histopathology, management aims, treatments offered and outcomes of these interventions were sought. Mode of presentation was defined as elective if surgery and treatment was planned and arranged, or, emergency if patient underwent hospital admission for complications including large bowel obstruction, perforation or life threatening haemorrhage. Follow up of all patients was performed in order to identify recurrence rates. Tumour staging was recorded using the American Joint Committee on Cancer Staging System (AJCC) which supersedes Dukes Classification. ${ }^{14}$ Right-sided lesions were defined as those from the cecum up to and including 
the hepatic flexure with transverse colon lesions recorded separately. Left-sided lesions included the splenic flexure and the descending colon but not the rectosigmoid junction. In cases of synchronous tumours, patients would be classified as multiple and not according to individual tumour location. Decisions on outcomes including stage of management (e.g. whether in remission or having progression etc) were based on whether treatment was curative or palliative in nature and were determined by discussions within an MDT setting.

Permission for this study was granted by the head of the Oncology department Dr Tigeneh Wondemagagnehu and the Dean of the University of Addis Ababa Medical School, Dr Daniel Siefu.

\section{Results}

A total of 95 CRC cases were identified during the study period of which 85 were eligible for analysis (age range 16-75) with the remainder excluded due to coding errors (Table 1). 44 patients were $\leq 40$ years old (young-onset) with $73 \%$ between 30-39years and 41 patients $>40$ years old (traditional-onset) with a Male: Female ratio of $1: 1$ and 1.41:1 respectively. Results are reported here as young/ traditional-onset disease. Pathological staging was reported in $94 \%$ $(\mathrm{n}=80)$ with pT3 staging the most commonly reported $(50 \% / 51 \%)$ with $30 \%$ of young-onset cases exhibiting perineural/lymphovascular involvement. Appropriate nodal Assessment (>12 nodes) at biopsy was low having been performed in only $59 \% / 56 \%$ of patients. Clinically, young-onset patients were more likely to present within 6 months of symptom onset $(53 \% \mathrm{v} 39 \%)$ with $16 \%$ traditional-onset CRC patients presenting $\geq 24$ months (range $24-39$ months). Comorbid illness featured in $76 \%$ and $55 \%$ of traditional-onset and young-onset cases respectively. Of traditional-onset patients $33 \%(\mathrm{n}=10)$ had features of the metabolic syndrome while $15 \%(n=3)$ young-onset had HIV. Performance status in the majority was ECOG 0/1 (77\%/83\%) with young-onset patients more likely to suffer functional limitation and were the only cases of ECOG V recorded $(n=2)$. Family History featured in $9 \%$ and $5 \%$ of young and traditional-onset patients respectively however in the majority of individuals no history was recorded $(52 \% \mathrm{v} 51 \%)$. In all patients with positive family history the presence of hereditary cancer syndromes was excluded.

\section{Tumour histopathology}

All patients had histological data reported with adenocarcinoma the most common histological subtype $(84 \% \mathrm{v} 85 \%)$ of which a history of polyps was only recorded in $3.5 \%$ of all patients (Figure 1). $72 \%$ of young-onset adenocarcinoma diagnoses $(n=31)$ were either moderately or poorly differentiated in nature with $60 \%$ of poorly differentiated tumours involving the rectum versus $42 \%$ traditionalonset patients $(\mathrm{n}=17)$ who exhibited poor differentiation and advanced stage. Rare histology's were confirmed in both cohorts, with similar levels of Mucinous Cell histology recorded $11 \%(n=5) / 10 \%(n=4)$. However, only in young-onset patients was Signet Ring histology present (5\%) with $50 \%$ of these patients exhibiting metastases.

\section{Presenting symptoms}

Young-onset patients most commonly presented with symptoms of abdominal pain $41 \%(n=18)$ and rectal bleeding $18 \%(n=8)$ (Figure 2). All cases of rectal bleeding and discharge symptoms were found in rectosigmoid cancers with $50 \%$ of those experiencing abdominal pain suffering right-sided malignancy. Emergency presentation was more common in young-onset patients $(16 \% / 10 \%)$ with bowel obstruction the most common presentation, $86 \%$ of these occurred in left sided malignancy. Traditional-onset patients $(n=39)$ had varied presentation with Rectal bleeding $(\mathrm{n}=9)(23 \%)$, abdominal pain $(\mathrm{n}=8)(21 \%)$ and change in bowel habit $(n=9)(23 \%)$ most prevalent. However, while of low incidence, traditional-onset patients exhibited higher incidence of abdominal mass $(8 \% \mathrm{v} 2 \%)$ which in $66 \%$ cases was associated with right-sided malignancy and longer duration of symptoms ( $>6$ months). Sole weight loss was an uncommon presenting symptom. However some weight loss was noted in $87 \%$ of all patients. Traditional-onset patients were more likely to present with significant weight loss $(>10 \%)(56 \% / 31 \%)$.

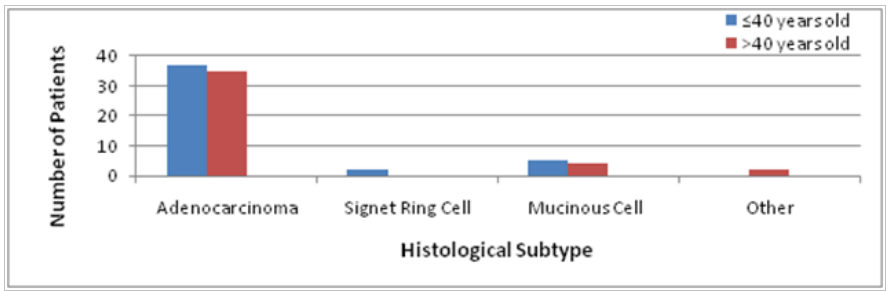

Figure I Histological Subtype recorded at Biopsy amongst Young $(n=44)$ and Traditional-onset $(n=4 I)$ CRC cases.

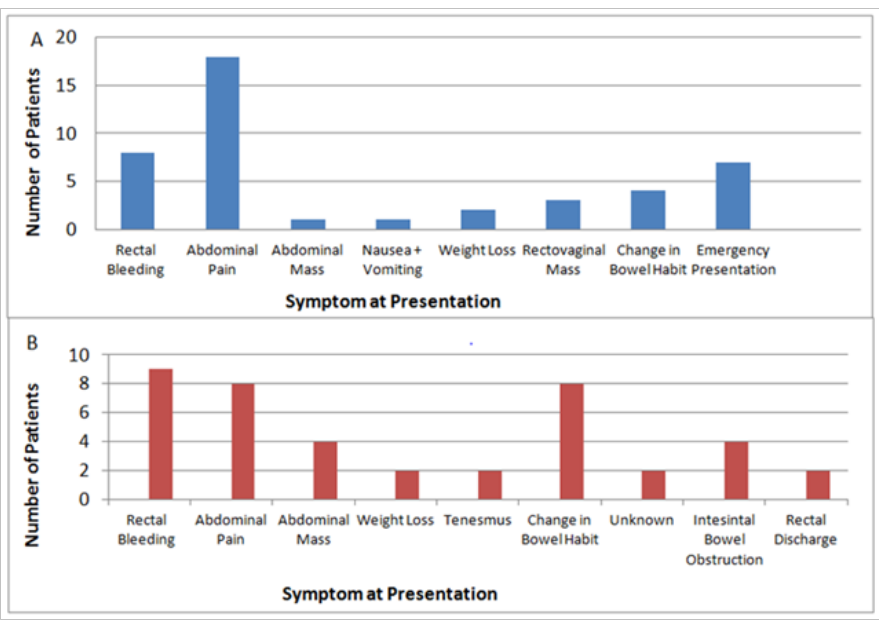

Figure 2 Presenting symptoms in CRC patients designated as (A) Youngonset and (B) Traditional-onset CRC.

\section{Tumour location}

Rectal cancer was the most common tumour sub-site in both cohorts although to a greater degree in traditional-onset patients $(39 \% \mathrm{v} 54 \%)$. Young-onset individuals were more predisposed to rightsided tumours $(22.73 \% \mathrm{v} 17.07 \%)$ and were the only cases of transverse colon involvement (5\%) and multiple/synchronous tumours (4.55\%). Compounding these results in the young was that $82 \%$ of patients presented at pathological stage T3/T4 compared to $76 \%$ in those $>40$ years, suggesting inherently more invasive disease (Figure 3 ).

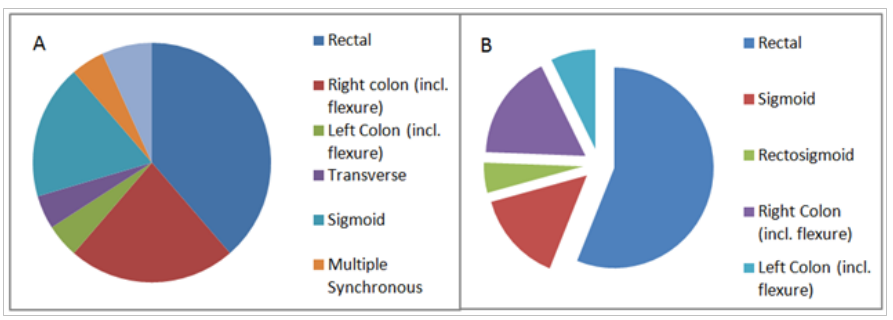

Figure 3 Tumour anatomical sub-site according to age designation (A) $\leq 40$ years old $(B)>40$ years old. 


\section{Baseline CEA levels}

Baseline CEA levels (Normal 0-5ng/mL) were measured in $95 \%$ $(42 / 44)$ and $78 \%$ (32/41) of young and traditional-onset patients respectively (Figure 4). In both cohorts there was heterogeneity. However this was more present within young-onset patients by virtue of the widened box plot (IQR=12.9 v 6.6). In our young-onset cohort data was negatively skewed with the median of young-onset being recorded as normal $(3.08 \mathrm{ng} / \mathrm{mL})$. In contrast in our traditional-onset cohort the data was positively skewed with median $(2.74 \mathrm{ng} / \mathrm{mL})$ and it was in this cohort that non-reactive samples were recorded in histopathologically advanced cases. In both cohorts major outliers existed $(518.58$ and $874 \mathrm{ng} / \mathrm{mL})$.

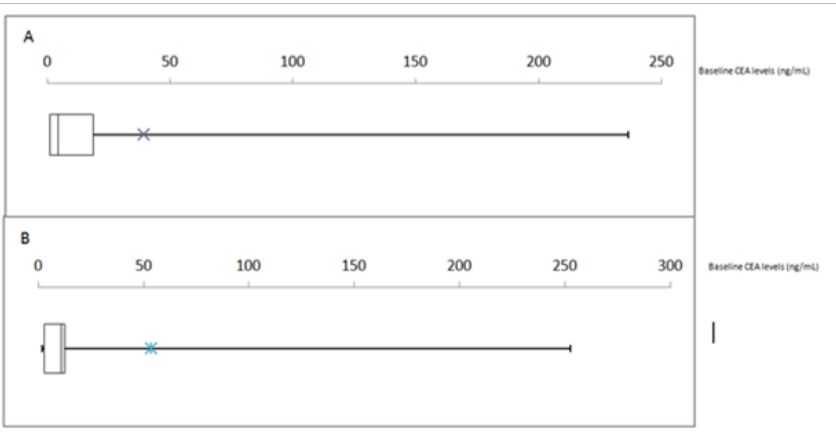

Figure 4 Box-Whisker Plots of Baseline Cancer Embryonic Antigen (CEA) levels in (A) $\leq 40$ year old cohort and (B) $>40$ year old cohort $(n=4$ I/44 and $33 / 4$ I respectively). *Represents mean CEA values.

\section{Treatment and outcome}

$87 \%(74 / 85)$ of all patients received standard treatment of which
$56.5 \%(48 / 85)$ were managed with curative intent and were equally distributed between young and traditional-onset groups. Young-onset patients were more likely to be enrolled in palliative management programmes $(43 \% / 30 \%)$ and of these $69 \%$ had rectosigmoid cancer and $37 \%$ pT4 staging. Of those patients managed curatively $98 \%$ (46/48) received at least one surgical intervention, and of these $96 \%$ received adjuvant chemotherapy. The most common regimen used was 5-Flurouracil+Calcium Leucovorin $(\mathrm{n}=19)$ followed by FOLFOX $(n=15)$ with multiple regimens recorded in $\sim 20 \%(n=9)$. Of rectal cancer patients' $\leq 40$ years only $41 \%(7 / 17)$ had completed treatment that included radiotherapy while only $36 \%$ traditional-onset patients had radiotherapy as indicated.

At the time of the analysis $2(2 \%)$ had died, 27 were in remission (32\%), $20(24 \%)$ were undergoing treatment and 36 had progressive disease $(42 \%)$ of those $\leq 40$ years with progressive disease were more likely to have rectosigmoid cancer (68\%), with higher levels youngonset patients $(34 \%)$ in remission compared to traditional-onset patients (24\%). Through long term follow-up of all patients (average 12 months) the incidence of recurrence was recorded. In both groups similar recurrence rates were noted $(15 \% / 16 \%)$. In young-onset patients with recurrence 5/6(83\%) were in Female patients with rare histology, Rectosigmoid/Right-sided malignancy $(50 \% / 50 \%)$, and emergency presentation $2 / 6(33 \%)$. Outcomes of treatment revealed that patients $>40$ years were most likely to experience complications $(n=26)$ (Table 2). Traditional-onset patients were more likely to suffer post-operative wound infection (15\%), urinary retention (19\%) and were the only instances of Incisional hernia (12\%). In youngonset cases with treatment complications $29 \%$ had an oncological emergency of which Neutropenic Sepsis $(75 \%)$ was most common. In both cohorts low levels of Surgical Revision and Postoperative Nausea and Vomiting.

Table I Baseline characteristics of 85 CRC patients divided by age as young ( $\leq 40$ years old) $(n=44)$ and Late $(>40$ years old) onset $(n=4 I)$

\begin{tabular}{|c|c|c|c|}
\hline & All CRC Patients $(n=85)$ & young-onset CRC $(n=44)$ & traditional-onset CRC $(n=4 I)$ \\
\hline Age Mean (years) & 43.6 & 33.2 & 55.2 \\
\hline 29-Oct & $12(13)$ & $12(27)$ & $0(0)$ \\
\hline $30-49$ & $43(5 \mathrm{I})$ & $32(73)$ & $\mathrm{II}(27)$ \\
\hline $50-69$ & $26(31)$ & $0(0)$ & $26(63)$ \\
\hline $70+$ & $4(5)$ & $0(0)$ & $4(10)$ \\
\hline \multicolumn{4}{|l|}{ Gender (\%) } \\
\hline Male & $46(54)$ & $22(50)$ & 24(59) \\
\hline Female & $39(46)$ & $22(50)$ & $17(4 \mid)$ \\
\hline \multicolumn{4}{|l|}{ Region (\%) } \\
\hline Addis Ababa & $56(66)$ & $24(55)$ & $32(78)$ \\
\hline Oromiya & $14(16)$ & $7(16)$ & $7(17)$ \\
\hline Snnpr & $6(7)$ & $5(11)$ & $\mathrm{I}(2.5)$ \\
\hline Other & $9(11)$ & $8(18)$ & $\mathrm{I}(2.5)$ \\
\hline \multicolumn{4}{|c|}{ Stage at Diagnosis (\%) } \\
\hline TI & $2(2)$ & $\mathrm{I}(2)$ & $\mathrm{I}(2)$ \\
\hline $\mathrm{T} 2$ & $12(14)$ & $6(14)$ & $6(15)$ \\
\hline T3 & $44(52)$ & $23(5)$ & $2 I(5 I)$ \\
\hline T4 & $22(26)$ & $13(30)$ & $9(22)$ \\
\hline N/A & $5(6)$ & $I(2)$ & $4(10)$ \\
\hline \multicolumn{4}{|c|}{ Nodal Involvement (\%) } \\
\hline NX & $36(42)$ & $\mid 8(4 I)$ & $18(44)$ \\
\hline No & $17(20)$ & $8(18)$ & $9(22)$ \\
\hline
\end{tabular}


Table continued...

\begin{tabular}{|c|c|c|c|}
\hline & All CRC Patients $(n=85)$ & young-onset CRC $(n=44)$ & traditional-onset CRC $(n=4 I)$ \\
\hline $\mathrm{NI}$ & $13(15)$ & $9(20)$ & $4(10)$ \\
\hline N2 & $8(9)$ & $6(14)$ & $2(5)$ \\
\hline N3 & $\mathrm{I}(\mathrm{I})$ & $0(0)$ & $\mathrm{I}(2)$ \\
\hline N4 & $3(4)$ & $2(5)$ & $\mathrm{I}(2)$ \\
\hline$N / A$ & $6(7)$ & $\mathrm{I}(2)$ & $5(12)$ \\
\hline \multicolumn{4}{|c|}{ Symptom Duration(\%) } \\
\hline $0-6$ months & $38(45)$ & $23(52)$ & $15(37)$ \\
\hline $6-12$ months & $18(21)$ & $7(16)$ & II (27) \\
\hline I2-24 months & $16(19)$ & $10(23)$ & $6(15)$ \\
\hline $24+$ months & $9(11)$ & $3(7)$ & $6(15)$ \\
\hline Unknown & $4(4)$ & $\mathrm{I}(2)$ & $3(7)$ \\
\hline \multicolumn{4}{|c|}{ Presence of Co-morbid IIIness (\%) } \\
\hline Yes & $51(60)$ & $20(45)$ & $30(73)$ \\
\hline No & $34(40)$ & $24(55)$ & $\mathrm{II}(27)$ \\
\hline \multicolumn{4}{|c|}{ ECOG status (\%) } \\
\hline 0 & $9(11)$ & $2(5)$ & $7(17)$ \\
\hline I & $59(69)$ & $32(72)$ & $27(66)$ \\
\hline II & $8(9)$ & $5(I I)$ & $3(7)$ \\
\hline III & $3(4)$ & $I(2)$ & $2(5)$ \\
\hline IV & $0(0)$ & $0(0)$ & $0(0)$ \\
\hline V & $2(2)$ & $2(5)$ & $0(0)$ \\
\hline$N / A$ & $4(5)$ & $2(5)$ & $2(5)$ \\
\hline \multicolumn{4}{|c|}{ Family History (\%) } \\
\hline Yes & $6(7)$ & $4(9)$ & $2(5)$ \\
\hline No & $35(4 I)$ & 17(39) & $18(44)$ \\
\hline N/A & $44(52$ & $23(52)$ & $2 I(5 I)$ \\
\hline
\end{tabular}

Were noted (5\%v4\%).

Table 2 Complication of Treatment and Disease Process

\begin{tabular}{|c|c|c|}
\hline Nature of complication & $\leq 40$ years $(n=21)(\%)$ & $>40$ years $(n=26)(\%)$ \\
\hline Post-op wound infection & $2(10)$ & $4(15)$ \\
\hline Surgical Revision & $I(5)$ & $\mathrm{I}(4)$ \\
\hline Oncological Emergency & $6(29)$ & $3(12)$ \\
\hline Fistula & $3(14)$ & $2(8)$ \\
\hline Bowel Obstruction & $7(33)$ & $4(15)$ \\
\hline Infection at other Site & $2(10)$ & $\mathrm{I}(4)$ \\
\hline \multicolumn{3}{|l|}{ Other } \\
\hline Incisional Hernia & $0(0)$ & $3(12)$ \\
\hline Anemia & $3(14)$ & $0(0)$ \\
\hline Urinary Retention/ Incontinence & $4(19)$ & $6(23)$ \\
\hline Poor Resection Margins & $2(10)$ & $2(8)$ \\
\hline Post-operative Nausea/Vomiting & $\mathrm{I}(5)$ & $\mathrm{I}(4)$ \\
\hline
\end{tabular}

\section{Discussion}

Young-onset CRC may represent a clinicopathologically distinct tumour associated with altered outcomes. Its appearance in developing countries has been attributed to westernization of diet and lifestyle, as it has the population structures of said nations which restrict disease in the young. ${ }^{15}$ Nevertheless, up to $62 \%$ of the projected increases in worldwide CRC cases over the next 20years will be in developing nations. ${ }^{16}$
In keeping with a recent systematic review, young-onset $\mathrm{CRC}$ was defined here as $\leq 40$ years-old. ${ }^{17} 52 \%$ of our patients were 'young-onset' which is far higher than the traditionally quoted $7-10 \%$ of western cohorts, and is consistent albeit at a higher proportion than previous reported work from the region. ${ }^{17,18}$ This difference can be explained by inherent institutional bias and the improved routine use of colonoscopy and advanced medical imaging at this centre. While young-onset CRC has previously been associated with delayed presentation, attributed to superior tolerance of symptoms, low consultation rates and physician 
misdiagnosis, no appreciable delay was found here with $52 \%$ of our young-onset patients presenting within 6 months of symptom onset although as $86 \%$ had pT3/pT4 staging perhaps more symptomatic disease and therefore earlier presentation. However, as $23 \%$ of youngonset patients presented in the $12-24$ month range this would suggest patient education of CRC presentation remains an important issue.

Unlike the more varied presentation of traditional-onset patients that included change in bowel habits and significant weight loss, young-onset patients more commonly suffered abdominal pain (41\%) and emergency presentation (16\%)the latter of which is linked to both poor short and long term outcomes and more invasive disease. ${ }^{4}$. This preponderance for abdominal pain in young-patients is thought to reflect the effects of ageing on nociceptive afferent fibres and their subsequent desensitisation to neurochemical and mechanical input. ${ }^{19}$. As such chronic abdominal pain in the context of other CRC symptoms in the young should raise the possibility of CRC even when functional limitations do not exist.

Surprisingly, a large percentage of our young-onset cohort (55\%) reported co-morbid disease with HIV present in 15\% (Ethiopia HIV prevalence-1.14\%). ${ }^{20}$ HIV has been epidemiologically linked to increased risk of CRC; although Kaposi sarcoma of the Bowel presents similarly. ${ }^{21}$ As such surveillance of these patients is prudent. In contrast, traditional-onset patients were more likely to have features of the metabolic syndrome. This is important as each 5 unit increase in BMI increases CRC risk by $30 \%$ while type 2 Diabetes Mellitus increases risk by $29 \%$ and $34 \%$ in men and women respectively. ${ }^{22}$ This indicates the need for improved preventative and management measures for these metabolic conditions in Ethiopia. Identifying at risk young-onset patients is challenging and our data remains inconclusive as to the importance of family history of CRC as this data was unavailable in the majority. Future work should try to relate the age and tumour characteristics of our young-onset CRC patients and their relatives to see if the principles of genetic anticipation exist whereby the presence and severity of symptoms presents earlier in successive generations. Good practice should include surveillance of young-family members of CRC patients in this setting with all youngonset cases receiving genetic testing to exclude familial syndromes which currently is often omitted. ${ }^{23}$

Histopathologically our young-CRC cases had a high incidence of right-sided/rectal tumours, perineural/lymphovascular invasion, nodal involvement, moderate/poor differentiation and metastatic disease which reflects recent reports. ${ }^{11}$ Mucinous CRC which is favourable in non-metastatic non-rectal disease was reported here at similar levels to western populations (11\%). Signet-ring histology however was found more common here than in western cohorts $(5 \% \mathrm{v} 1 \%)$ and was associated with metastasis and poor outcomes which others have reported. ${ }^{24}$. Both these histology's and others are linked to underlying microsatellite instability and suggest overlap with Lynch syndrome. ${ }^{25}$ While the above features are implicated in poor survival, ethnicity is also a determinant of prognosis with black people having the worst prognosis. ${ }^{26}$

It is important to note however the limitations of this work such as the centres use of multiple private sources for histopathology reporting and tumour biochemistry (CEA). As such standardization of measuring and reporting these results could influence our findings. For instance nodal staging requires $\geq 12$ lymph nodes if it is to avoid understaging which may lead to erroneous use/disuse of neoadjuvant/ adjuvant therapy, ${ }^{27}$ however this practice was underperformed in our cohort $(59 \% / 56 \%)$. In addition CEA a prognostic tumour marker at baseline and a method for detecting recurrence showed great heterogeneity and asymmetry between our cohorts. ${ }^{28}$ Indeed $44 \%$ $(37 / 85)$ of our patients had normal baseline levels. This variability and normalization of CEA values may be explained by differential production by anatomical subsite, different methods of its detection, and its sensitivity to benign processes (e.g. smoking). Other limitations include the retrospective analysis of paper health records which were often incomplete or misplaced as well as poor patient educational attainment which may have influenced some of the results reported here. While no statistical analyses were performed we do believe these results have merit in highlighting the different behaviours and outcomes of CRC in Ethiopia by age of onset. The outcome of young-onset CRC remains controversial with studies limited by their small population size. It is thought that despite their predisposition to advanced disease they may still have a better prognosis in low-stage non-metastatic disease. ${ }^{15,29}$

While determinations regarding survival cannot be made by our data, by the time of analysis two young-onset patients had died and overall a similar rates of recurrence versus traditional-onset patients was recorded (15\%/16\%) which is concerning and suggests suboptimal treatment. Young-onset patients showed a greater likelihood for palliative management (43\%) with $69 \%$ of these patients suffering rectosigmoid diseases. This would suggest that rectal disease in this cohort is inherently more hazardous and may be of a different biology and outcome versus other anatomical subsites. Indeed while treatment provision was high overall $(87 \%)$ only $41 \% / 36 \%$ patients of our rectal cancer cohort had appropriate neoadjuvant radiotherapy. Radiotherapy and Total Mesorectal Excision (TME) are considered cornerstones of curative treatment in advanced rectal disease. ${ }^{30}$ While TME was not performed at this centre access to treatment remains an issue with the current radiotherapy waiting list exceeding one year. Moreover, due to drug unavailability $20 \%$ of all patients on chemotherapy experienced cycle-interruptions which can yield resistant subclones and increase long-term recurrence and mortality rates.

However as with any treatment a benefit/harm ratio must be considered. While young-onset patients were less likely to suffer postop-wound infection and surgical complications they did have a high incidence of post-chemotherapeutic febrile neutropenia. This would suggest young-patients are affected greater by current regimes as a result of more invasive underlying disease and higher levels of anaemia and are both risk factors for its development. ${ }^{31}$ Due to the great risk of mortality with this complication greater surveillance and use of preventative measures in this cohort may be required than in traditional oncological populations.

To conclude in this Ethiopian based study we found high levels of young-onset CRC of which rectal disease was the most prevalent. These patients often had poor histopathological factors such as advanced staging, lymphovascular/perineural involvement and mucinous/signet-ring histology resulting in a high likelihood of palliative management. With this in mind we suggest that young-onset CRC does not behave in the same manner as traditional traditionalonset CRC and showed a trend to inferior prognosis. Much work remains in understanding the pathogenesis of this condition in order to identify potential risk factors and establish new biomarkers that may help identify cases and predict therapeutic responses as there is a high level of treatment complications.

\section{Acknowledgements}

None. 


\section{Conflicts of interest}

The authors declare that there are no conflicts of interest.

\section{Funding}

None.

\section{References}

1. She KM, Wang HM, Chen JB, et al. Colorectal Cancer in Younger than 30 Years Old Group is not Associated with Poor Prognosis. J Soc Colon Rectal Surgeon. 2011;22:93-98.

2. Bretthauer M. Evidence for Colorectal Cancer Screening. Best Practice\& Research Clinical Gastroenterology. 2010;24:417-425.

3. Van Steenbergen LN, Lemmens VE, Louwman MJ, et al. Increasing incidence and decreasing mortality of colorectal cancer due to marked cohort effects in southern Netherlands. Eur J Cancer Prev. 2009;18(2):145-152.

4. Mansouri D, McMillan DC, Crearie C, et al. Temporal trends in mode, site and stage of presentation with the introduction of colorectal cancer screening:a decade of experience from the West of Scotland. British Journal of Cancer. 2015;113(3):556-561.

5. Arnold M, Sierra MS, Laversanne M, et al. Global patterns and trends in colorectal cancer incidence and mortality. Gut. 2016.

6. Jinjuvadia R, Lohia P, Jinjuvadia C, et al. The association between metabolic syndrome and colorectal neoplasm systemic review and meta-analysis. J Clin Gastroenterol. 2013;47(1):33-44.

7. Brenner H, Hoffmeister M, Stegmaier C et al. Risk of progression of advanced adenomas to colorectal cancer by age and sex:estimates based on 840,149 screening colonoscopies. Gut. 2007;56:1585-1589.

8. Wang R, Wang MJ, Ping J. Clinicopathological Features and Survival Outcomes of Colorectal Cancer in Young Versus Elderly. Medicine. 2015;94(35):1-8.

9. Chou CL, Chang SC, Lin TC, et al. Differences in clinicopathological characteristics of colorectal cancer between younger and elderly patients: an analysis of 322 patients from a single institution. The American Journal of Surgery. 2011:202:574-582.

10. Stigliano V, Sanchez-Mete L, Martayan A, et al. Early-onset colorectal cancer:A sporadic or inherited disease? World $J$ Gastroenterol. 2014;20(35):12420-12430.

11. Ballester V, Shahrooz Rashtak, et al. Clinical And molecular features of young-onset colorectal cancer. World J Gastroenterol. 2016;22(5):17361744 .

12. Silla I, Rueda D, Rodriguez Y, et al. Early-onset colorectal cancer:A separate subset of colorectal cancer. World $J$ Gastroenterol. 2014;20(46):17288-17296.

13. Goldberg J, Furman WL. Management of Colorectal Carcinoma in Children and Young Adults. Journal of Paediatric Haematology/ Oncology. 2012;34:76-79.
14. Halim I. Essential Revision Notes in Surgery for Medical Students. Pas Test. 2008.

15. Jones GH, Radwan R, Davies M, et al. Clinicopathological characteristics of colorectal cancer presenting under the age of 50. International Journal of Colorectal Disease. 2015;30:483-489.

16. Pourhoseingholi MA. Epidemiology and burden of colorectal cancer in Asia-Pacific region:what shall we do now ? Translational Gastrointestinal Cancer. 201;3(4).

17. O'Connell JB, Maggard MA, Livingston EH, et al. Colorectal cancer in the young. Am J Surg. 2004;187(3):343-348.

18. Ashenafi S. The frequency of large bowel cancer as seen in Addis Ababa University, Pathology Department. Ethiop Med J. 2000;38(4):277-282.

19. de Sousa JB1, Souza CS, Fernandes MB, et al. A Do young patients have different clinical presentation of colorectal cancer causing delay in diagnosis? Int J Colorectal Dis. 2014;29(4):519-527.

20. World Health Organisation. Ethiopia- HIV/AIDs.

21. Ford RM, McMahon MM, Wehbi MA. HIV/AIDS and Colorectal Cancer: A review in the era of antiretrovirals. Gastroenterol Hepatol (NY). 2008;4(4):274-278.

22. Trabulo D, Ribeiro S, Martins C, et al. AMetabolic Syndrome and colorectal neoplasms: An ominous association. World J Gastroenterol. 2015;21(17):5320-5327.

23. Stoffel EM. Colorectal Cancer in Young Individuals: Opportunities for Prevention. Journal of Clinical Oncology J Clin Oncol. 2015;33 (31):3525-3527.

24. Nagtegaal ID, Hugen N. The Increasing Relevance of Tumour Histology in Determining Oncological Outcomes in Colorectal Cancer. Curr Colorectal Cancer Rep. 2015;11(5):259-266.

25. Prognostic Impact of Microsatellite Instability in Colorectal Cancer Presenting With Mucinous, Signet-Ring and Poorly Differentiated Cells. Ann Coloproctol. 2016;32(2):58-65.

26. Holowatyi AN, Ruterbusch JJ, Rozek LS, et al. ARacial/Ethnic Disparities in Survival Among Patients With Young-Onset Colorectal Cancer. J Clin Oncol. 2016;34(18):2148-2156.

27. Wong SL. Lymph node counts and survival rates after resection for colon and rectal cancer. Gastrointest Cancer Res. 2009;3(2):33-35.

28. Liu Z, Zhang Y, Niu Y, et al. AA systematic Review and Meta-Analysis of Diagnostic and Prognostic Serum Biomarkers of Colorectal Cancer. PLoS ONE. 2014;9(8):e103910.

29. Li Q, Cai G, Li D, Wang Y, et al. ABetter Long Term Survival in Young Patients with Non-Metastatic Colorectal Cancer after Surgery, an Analysis of 69,835 Patients in SEER Database. PLoS ONE. 2017;9(4):e93756.

30. Milinis K, Thornton M, Montazeri A, et al. Adjuvant chemotherapy for rectal cancer: Is it needed? World J Clin Oncol. 2015;6(6):225-236.

31. Lyman GH, Abella E, Pettengell R. Risk factors for febrile neutropenia among patients with cancer receiving chemotherapy:A systematic review. Crit Rev Oncol Hamatol. 2014;90(3):190-199. 